\title{
Socio-demographic characteristics associated with HIV and syphilis seroreactivity among pregnant women in Blantyre, Malawi, 2000-2004
}

\author{
Jesse J. Kwiek ${ }^{1,6}$, Victor Mwapasa ${ }^{2,6}$, Alisa P. Alker ${ }^{1}$, Adamson S. Muula ${ }^{1,2}$, Humphreys E. Misiri ${ }^{2}$, \\ Malcolm E. Molyneux ${ }^{3,4}$, Stephen J. Rogerson ${ }^{5}$, Frieda M. Behets ${ }^{1}$, Steven R. Meshnick ${ }^{1}$
}

1. Department of Epidemiology, University of North Carolina at Chapel Hill, Chapel Hill, North Carolina, USA.

2. Division of Community Health, College of Medicine, Blantyre, Malawi.

3. Malawi-Liverpool-Wellcome Trust Clinical Research Programme, College of Medicine, Blantyre, Malawi

4. School of Tropical Medicine, University of Liverpool, Liverpool UK.

5. Department of Medicine, University of Melbourne, Parkville, Victoria, Australia.

6. These authors contributed equally to this manuscript.

Corresponding author: Jesse J. Kwiek, Center for Microbial Interface Biology, The Ohio State University, 1008 Biological Research Tower, 460 W. 12th Ave., Columbus, OH 43210, (614) 292-3256, (614) 292-9616 (fax), email: kwiek.2@osu.edu

\section{Abstract}

Objectives: We aimed to evaluate socio-demographic factors associated with HIV and syphilis seroreactivity in pregnant Malawians presenting for antenatal care in late third trimester of pregnancy.

Methods: Between December 2000 and March 2004 at Queen Elizabeth Central Hospital Blantyre, Malawi, we collected cross-sectional clinical and socioeconomic data from consenting women. HIV-1 status was determined using rapid HIV antibody tests and syphilis seroreactivity was determined using Rapid Plasma Reagin (RPR) and confirmed with Treponema pallidum hemagglutination assay (TPHA).

Results: Of 3,824 women screened for HIV, $1156(30 \%)$ were HIV seropositive and 198 (5\%) were RPR and TPHA seroreactive. In the multivariate analysis, HIV infection was positively associated with elevated socio-economic status, being formerly married, and age, but not with education level. HIV prevalence was lower in women of Yao ethnicity than in other women (OR: 0.78, 95\%CI: 0.64 - 0.95). Increased maternal education was negatively associated with syphilis seroreactivity.

Conclusions: The seroprevalence of HIV and syphilis among women attending the antenatal ward in Blantyre remains unacceptably high. Demographic correlates of HIV and syphilis infections were different. Our results demonstrate the need for better strategies to prevent HIV and syphilis in women and calls for optimizing antenatal syphilis screening and treatment in Malawi.

\section{Introduction}

Two thirds of all HIV/AIDS infected persons and 75\% of all HIV infected women live in Sub-Saharan Africa (SSA). Malawi, one of the countries in SSA most affected by HIV, reported a national HIV prevalence of $20 \%$ among pregnant women in $2003^{1}$. In addition to the prevalent HIV infections, in 2003, approximately 3\% of pregnant Malawian women were also infected with syphilis ${ }^{1}$. Syphilis is an important cause of maternal morbidity, still births, low birth weight and congenital malformations ${ }^{2-4}$. We have recently shown that maternal syphilis increases the risk of mother to child

\section{HIV transmission ${ }^{5}$.}

Relatively few people in SSA have access to HIV and syphilis control measures. A survey of 22 sub-Saharan countries showed that on average, only $38 \%$ of women who attended ANCs were screened for syphilis ${ }^{6}$; more recently, a communitybased survey in Western Kenya revealed that only 19\% of women attending ANC were tested for syphilis ${ }^{7}$. Because of significant health system constraints in Malawi, coverage of HIV and syphilis interventions is expected to remain low in the short-medium term ${ }^{8}$. In Malawi in 2005, it was estimated that only $3 \%$ of $\mathrm{HIV}$-infected pregnant women received nevirapine to reduce HIV mother-to-child transmission ${ }^{8}$. In order to better understand the epidemiology of HIV and syphilis among pregnant women in Malawi, we assessed the socio-demographic correlates of these two infections at an antenatal ward in Blantyre, Malawi.

\section{Methods}

\section{Setting}

Women seeking care at the Queen Elizabeth Central Hospital $(\mathrm{QECH})$ antenatal ward (ANW) in Blantyre, Malawi, from December 2000 until March 2004 were evaluated. QECH is the main tertiary referral hospital in southern Malawi and also functions as the district hospital for Blantyre; it handles approximately 15,000 deliveries per year. In general, pregnant women in Malawi attend prenatal care 16 to 20 weeks into the pregnancy, soon after quickening begins (fetal kicking).

\section{Study recruitment}

Women admitted to the ANW were screened and if eligible and consenting, enrolled in a study on malaria and mother to child transmission of HIV. The study had two goals, 1) to document the socio-demographic features associated with HIV infection in pregnant women, and 2) to investigate the effects of placental malaria on HIV mother-to-child transmission. Enrollment details and the results from the longitudinal cohort study of the HIV-infected women have been described ${ }^{5}$. Although documenting syphilis cases was not a stated goal of the study, in accordance with the Malawi Standard Treatment Guide, participants were tested serologically for syphilis as described below. Owing to logistical constraints and the short time period between enrollment and delivery (median 4.5 days), syphilis testing was done in batches, and as a result, many of the syphilisseroreactive women and their children were treated with benzathine penicillin postnatally.

A standard questionnaire was used to collect sociodemographic data. Marital status was categorized as single, married, or formerly married (separated or divorced or widowed). The cost of materials used in the construction of the primary residence was considered a surrogate of 
socio-economic status, with mud and pole construction representing the least expensive materials, brick walls with a grass-thatched roof representing a moderate expense, and brick walls with iron sheets representing the most expensive materials. For married women, their husband's occupation was categorized into 21 informal categories according to the perceived similarity of the level of training required for that career. Additionally, the women were asked if they had ever been diagnosed with HIV, a sexually transmitted infection (STI), or tuberculosis (TB). Previous STIs, except HIV and syphilis, and TB infection, were not verified by medical tests or medical records.

\section{Laboratory tests}

HIV status was determined simultaneously with the Determine $^{\text {TM }}$ HIV-1/2 Rapid Antibody Test (Abbott Laboratories, IL, USA) and the SeroCard ${ }^{\mathrm{TM}} \mathrm{HIV}-1 / 2$ Rapid Test (Trinity Biotech Plc, Co Wicklow, Ireland); discordant results were resolved with the HIVSPOT HIV-1/2 Rapid Test (Genelabs Diagnostics, Singapore). Women were tested for syphilis using the Rapid Plasma Reagin test ([RPR], Omega Diagnostics, Alloa, Scotland), and all RPR reactive sera were tested with the Treponema pallidum Hemagglutination Assay ([TPHA], Omega Diagnostics, Alloa, Scotland). Clinical signs of syphilis or recent treatment were not evaluated, and women with a reactive RPR followed by a reactive TPHA were considered syphilis seroreactive. Hemoglobin concentration was measured using a hemoglobinometer (HemoCue AB, Ängelholm Sweden), with anemia defined as a hemoglobin concentration less than $11 \mathrm{~g} / \mathrm{dL}$. Peripheral malaria infection was assessed on thick blood films stained with Field's stain.

\section{Statistical methods}

Data were entered into Microsoft Access in duplicate, crosschecked, and analyzed with Stata v8.2 (StataCorp, College Station, TX, USA). The association between nominal categorical variables and infection was evaluated with the chi-squared test for independence; monotonic trends in ordinal categorical variables were tested with a chi-squared statistic for trend ("ptrend" module, Stata). Previous studies have suggested an inverse association between HIV status and Islamic faith, and therefore we decided a priori to test the association between HIV and belonging to the Yao tribe, the predominant Muslim tribe in Malawi. Prevalence odds ratios (ORs) of binary variables associated with HIV and syphilis infection were calculated from contingency tables and tested with a chi-squared statistic or Fisher's exact test. In order to determine the independent correlates of HIV and syphilis seroreactivity, demographic features associated with either HIV or syphilis in the univariate analysis $(p<0.1)$ were included in a multivariable model.

\section{Ethical considerations}

The study was approved by the Malawi College of Medicine Research and Ethics Committee and by the Institutional Review Boards at both the University of Michigan and the University of North Carolina at Chapel Hill. Informed consent was received from all participants.

\section{Results}

\section{Participant characteristics}

Of the 6184 women invited to participate in this study, $3,824(62 \%)$ were enrolled. Over the course of the study, the acceptance rate declined, with 881 out of $1257(70 \%)$ enrolled the first year, 1389 out of 2080 (67\%) enrolled the second year, 1201 out of 2137 (56\%) enrolled the third year, and 353 out of $700(50 \%)$ enrolled in the fourth year. No additional information was ascertained from the women who declined to participate. HIV prevalence was $27.9 \%$ in $2001,29.4 \%$ in $2002,32.5 \%$ in 2003 , and $32.7 \%$ in 2004; syphilis seroreactivity by year was $7.3 \%, 4.6 \%, 4.2 \%$, and $5.7 \%$ respectively.

Table 1 outlines the demographic characteristics of the participants. Overall, peripheral $P$. falciparum parasites were detected in $9 \%$ of the women, $2 \%$ percent of the women reported being previously diagnosed with $\mathrm{HIV}$, 5\% with a STI, and 3\% with TB. When asked, $22 \%$ of the women reported fever during the week preceding enrollment, $32 \%$ general body pains and $42 \%$ headache. The mean hemoglobin concentration was $11.1 \mathrm{~g} / \mathrm{dL}(\mathrm{SD}=1.9)$.

\section{Factors associated with HIV seroreactivity.}

Thirty percent of the 3,824 women were HIV positive, with the highest HIV prevalence among women aged 26-30 (Table 1). Women with HIV infection more often reported previous diagnoses of TB, STI or HIV, as well as fever the previous week (data not shown). HIV-infected women were also more likely to have anemia (OR: $2.5,95 \% \mathrm{CI}: 2.1,2.9), P$ falciparum peripheral parasitemia (OR: 1.3, 95\%CI: 0.99, 1.6), and syphilis (Table 1). HIV prevalence varied by gravidity, marital status, and the location of their primary residence; the highest prevalence was observed among multigravid, formerly-married, and urban women. Women who selfidentified as Yao had a significantly lower HIV prevalence than the women of all other ethnicities combined $(26 \%$ versus 31\%, OR: 0.78 , 95\% CI: 0.64, 0.95).

HIV prevalence was similar among all education strata, but women who were formally employed had a higher HIV prevalence compared to those who were unemployed, and women with the most expensive houses had a higher HIV prevalence than those in less expensive housing (Table I).

Married women, who represent $91 \%$ of the study population, were stratified into 21 groups according to their husband's occupation. HIV prevalence varied significantly by their husband's occupation, ranging from $20 \%$ (married to a farmer) to $28 \%$ (married to a non-skilled laborer) to $45 \%$ (married to a driver) $\left(\chi^{2}=65 ; \mathrm{df}=20, \mathrm{p}<0.001\right)$.

\section{Factors associated with Syphilis seroreactivity}

Of 3,805 women screened, 285 (8\%) were RPR reactive. Of 270 RPR reactive sera tested, 198 (73\%) were TPHA reactive, resulting in an overall 5\% syphilis seroreactivity. There was no association between syphilis seroreactivity and peripheral malaria (OR: $0.76,95 \%$ CI $0.40,1.3)$. Four $(2 \%)$ of the women with- and $44(1 \%)$ of the women without syphilis seroreactivity reported taking antibiotics in the two weeks prior to enrollment. Syphilis seroreactivity was $8 \%$ among HIV positive women and $4 \%$ among HIV negative women (OR: 2.1, 95\%CI: 1.5, 2.8). Of the 195 syphilis seroreactive women with data on ANC attendance, 194 had attended the ANC at least once (median= 4, range 0 , 11). Syphilis seroreactivity was associated with anemia (OR: 1.4, 95\% CI 1.0, 1.9), previous diagnosis of STI (OR: 5.3, 95\%CI: 3.5, 8.0), as well as rural residence, and multigravidity (Table 1) and was not associated with self-reported TB, HIV diagnosis, or fever in the week preceding enrollment (results 
Table 1 Socio-demographic characteristics associated with HIV or Syphilis seroreactivity

\begin{tabular}{|c|c|c|c|c|c|}
\hline & \multirow{2}{*}{$\begin{array}{l}\text { Enrollment (N=3824) } \\
\mathrm{n}(\%)\end{array}$} & \multicolumn{2}{|c|}{$\begin{array}{l}\text { HIV-infected } \\
(\mathrm{N}=3824)\end{array}$} & \multicolumn{2}{|c|}{$\begin{array}{l}\text { Syphilis Seroreactive } \\
(\mathrm{N}=3790)\end{array}$} \\
\hline & & $\mathrm{n}(\%)$ & OR $(95 \% \mathrm{Cl})$ & $\mathrm{n}(\%)$ & OR $(95 \% \mathrm{Cl})$ \\
\hline Overall & & $1156(30)$ & & $198(5)$ & \\
\hline \multicolumn{6}{|l|}{ Age (in years) } \\
\hline median (range) ${ }^{*}$ & $22(14-51)$ & & & & \\
\hline$<20$ & $985(26)$ & $159(16)$ & 1. & $35(4)$ & 1. \\
\hline $20-25$ & $1,605(42)$ & $540(34)$ & $2.6(2.2,3.2)$ & $108(7)$ & $2.0(1.3,2.9)$ \\
\hline $26-30$ & $603(16)$ & $257(43)$ & $3.9(3.0,4.9)$ & $35(6)$ & $1.7(1.0,2.7)$ \\
\hline $31-35$ & $283(7)$ & $110(39)$ & $3.3(2.4,4.5)$ & $11(4)$ & $1.1(0.56,2.2)$ \\
\hline$>35$ & $156(4)$ & $34(22)$ & $1.4(1.0,2.2)$ & $4(3)$ & $0.72(0.25,2.1)$ \\
\hline missing & $192(5)$ & & $\chi^{2}=160, p<0.0001$ & & $\chi^{2}=16, p=0.003$ \\
\hline \multicolumn{6}{|l|}{ Employment } \\
\hline not working & $3023(79)$ & $865(29)$ & 1. & $156(2)$ & 1. \\
\hline formally-employed & $715(19)$ & $260(36)$ & $1.4(1.2,1.7)$ & $39(6)$ & $1.1(0.74,1.5)$ \\
\hline missing & $86(2)$ & & $\chi^{2}=17, p<0.0001$ & & $\chi^{2}=0.13, p=0.7$ \\
\hline
\end{tabular}

\section{Completed Level of}

Education

\begin{tabular}{|l|l|l|l|l|l|}
\hline none & $357(9)$ & $111(31)$ & 1. & $25(7)$ & 1. \\
\hline 4 years primary & $769(20)$ & $257(33)$ & $1.1(0.9,1.5)$ & $54(7)$ & $1.0(0.61,1.6)$ \\
\hline 8 years primary & $1540(40)$ & $445(29)$ & $0.90(0.70,1.2)$ & $83(5)$ & $0.76(0.48,1.2)$ \\
\hline 2 years secondary & $528(14)$ & $158(30)$ & $0.95(0.71,1.3)$ & $18(3)$ & $0.47(0.25,0.87)$ \\
\hline 4 years secondary & $610(16)$ & $183(30)$ & $0.95(0.72,0.26)$ & $17(3)$ & $0.38(0.20,0.71)$ \\
\hline missing & $20(1)$ & $\chi_{(\text {(trend) }}^{2}=1.2, p=0.3$ & & $\chi_{(\text {trend) }}^{2}=17, p<0.0001$ \\
\hline
\end{tabular}

\section{Cost of Housing}

Material

\begin{tabular}{|c|c|c|c|c|c|}
\hline high & $2639(69)$ & $854(32)$ & 1. & $133(5)$ & 1. \\
\hline medium & $1057(28)$ & $266(25)$ & $0.70(0.60,0.83)$ & $61(6)$ & $1.2(0.85,1.6)$ \\
\hline low & $104(3)$ & $24(23)$ & $0.63(0.39,1.0)$ & $4(4)$ & $0.75(0.27,2.1)$ \\
\hline missing & $24(1)$ & & $\chi_{\text {(trend) }}^{2}=20, p<0.001$ & & $\chi_{(\text {(trend) }}^{2}=0.20, p=0.7$ \\
\hline \multicolumn{6}{|l|}{ Gravidity } \\
\hline 1 & $1401(37)$ & $269(19)$ & 1. & $44(3)$ & 1. \\
\hline $2-4$ & $1860(49)$ & $714(38)$ & $2.6(2.2,3.1)$ & $119(7)$ & $2.1(1.5,3.0)$ \\
\hline$>4$ & $562(15)$ & $173(31$ & $1.9(1.5,2.3)$ & $35(6)$ & $2.1(1.3,3.3)$ \\
\hline missing & $1(0)$ & & $\chi^{2}=140, p<0.0001$ & & $\chi^{2}=19, p=0.0001$ \\
\hline \multicolumn{6}{|l|}{ Marital Status } \\
\hline married & $260(7)$ & $1044(30)$ & 1. & $180(5)$ & 1. \\
\hline single & 3468 (91) & $60(23)$ & $0.69(0.51,0.94)$ & $12(5)$ & $0.87(0.48,1.6)$ \\
\hline formerly married & $84(2)$ & $49(58)$ & $3.3(2.1,5.1)$ & $6(7)$ & $1.4(0.60,3.2)$ \\
\hline missing & $13(0)$ & & $\chi^{2}=38, p<0.001$ & & $\chi^{2}=0.82, p=0.7$ \\
\hline \multicolumn{6}{|l|}{ Residence } \\
\hline urban & $2720(71)$ & $856(32)$ & 1. & $131(5)$ & 1. \\
\hline periurban & 509 (13) & $148(29)$ & $0.89(0.72,1.1)$ & $28(6)$ & $1.1(0.75,1.7)$ \\
\hline rural & $518(14)$ & $132(25)$ & $0.74(0.60,0.92)$ & $35(7)$ & $1.4(0.98,2.1)$ \\
\hline outside Blantyre & $49(1)$ & $11(22)$ & $0.63(0.32,1.2)$ & $3(6)$ & $1.3(0.39,4.2)$ \\
\hline missing & $29(1)$ & & $\chi^{2}=9.3, p=0.03$ & & $\chi^{2}=3.6, p=0.3$ \\
\hline
\end{tabular}

Row percentages equal the \% HIV or syphilis positive per strata. Statistics presented do not include missing data.

*One woman reported being 15 years old, but was later found to be 14 years old. 
Table 2 Multivariable regression of sociodemographic factors

independently associated with HIV infection or Syphilis seroreactivity

\begin{tabular}{|l|l|l|}
\hline & HIV infection & Syphilis infection \\
\hline Variable & $\begin{array}{l}\text { Adjusted OR } \\
(95 \% \mathrm{Cl})\end{array}$ & $\begin{array}{l}\text { Adjusted OR } \\
(95 \% \mathrm{Cl})\end{array}$ \\
\hline
\end{tabular}

Age (in years)

\begin{tabular}{|l|l|l|}
\hline$<20$ & 1. & 1. \\
\hline $20-25$ & $2.0(1.6,2.6)$ & $1.4(0.83,2.1)$ \\
\hline $26-30$ & $2.9(2.2,4.0)$ & $0.74(0.39,1.4)$ \\
\hline $31-35$ & $2.7(1.8,4.0)$ & $0.39(0.16,0.96)$ \\
\hline$>35$ & $1.5(0.91,2.6)$ & $0.17(0.05,0.65)$ \\
\hline
\end{tabular}

Completed Level of Education

\begin{tabular}{|l|l|l|}
\hline none & 1. & 1. \\
\hline 4 years primary & $1.2(0.91,1.7)$ & $0.92(0.55,1.5)$ \\
\hline 8 years primary & $1.0(0.78,1.4)$ & $0.68(0.42,1.1)$ \\
\hline 2 years secondary & $1.1(0.81,1.6)$ & $0.44(0.22,0.86)$ \\
\hline 4 years secondary & $1.1(0.75,1.5)$ & $0.35(0.17,0.71)$ \\
\hline
\end{tabular}

\section{Cost of Housing Material}

\begin{tabular}{|l|l|l|}
\hline high & 1. & 1. \\
\hline medium & $0.74(0.61,0.90)$ & $0.87(0.60,1.3)$ \\
\hline low & $0.78(0.46,1.3)$ & $0.47(0.14,1.6)$ \\
\hline Formal Employment & $1.2(1.0,1.5)$ & $1.2(0.78,1.7)$ \\
\hline \multicolumn{3}{|l|}{} \\
\hline \multicolumn{1}{|l|}{ Gravidity } & 1. & 1. \\
\hline 1 & $1.7(1.4,2.1)$ & $2.0(1.2,3.1)$ \\
\hline $2-4$ & $1.1(0.77,1.6)$ & $3.1(1.5,6.2)$ \\
\hline$>4$ &
\end{tabular}

\section{Marital Status}

\begin{tabular}{|l|l|l|}
\hline married & 1. & 1. \\
\hline single & $0.80(0.58,1.2)$ & $0.73(0.36,1.5)$ \\
\hline formerly married & $3.1(1.7,5.7)$ & $0.85(0.27,2.7)$ \\
\hline
\end{tabular}

\section{Member of Yao Tribe}

\begin{tabular}{|l|l|l|}
\hline no & 1. & 1. \\
\hline yes & $0.75(0.61,0.92)$ & $0.87(0.58,1.3)$ \\
\hline
\end{tabular}

\section{Residence}

\begin{tabular}{|l|l|l|}
\hline urban & 1. & 1. \\
\hline semi-urban & $0.86(0.68,1.1)$ & $1.2(0.74,1.8)$ \\
\hline rural & $0.81(0.63,1.0)$ & $1.3(0.82,2.1)$ \\
\hline
\end{tabular}

not shown). Syphilis seroreactivity was associated with a selfreported history of miscarriage or stillbirth (OR: $2.3,95 \% \mathrm{CI}$ : $1.6,3.5)$ and was most common among multigravid women in their early twenties (Table 2). Syphilis was not associated with maternal employment, cost of housing material, being a Yao, or marital status. Syphilis seroreactivity was inversely associated with maternal education $\left(\chi_{\text {(trend) }}^{2}=17, \mathrm{p}<0.0001\right)$.

\section{Factors associated with dual HIV and Syphilis seroreactivity}

Ninety-two $(2.4 \%)$ of the participants were seroreactive for both HIV and syphilis. Dual infection was most prevalent among $20-25$ year olds (3.6\%), and dual infection, like syphilis seropositivity, was inversely associated with education level. Dual infection was not associated with formal employment, residence, cost of housing material, or being a member of the Yao tribe. However, due to the small number of duallyinfected women, these results should be interpreted with caution.

\section{Factors associated with HIV versus Syphilis seroreactivity in multivariate analyses}

Table 2 shows the demographic variables that were independently associated with HIV and syphilis seroreactivity. HIV infection was independently associated with age 20 years or older, formal employment, gravidity between 2-4, high cost of housing materials, and former marriage, and it was inversely associated with Yao identity. Syphilis seroreactivity was independently associated with multigravidity and inversely associated with both age and education level.

\section{Discussion}

Among women who attended a tertiary-care hospital in urban Malawi in late pregnancy, HIV infection was positively associated with formerly being married, and multigravidity, but was not associated with the level of maternal education. Syphilis infection was independently and positively associated with multigravidity and was highest among women with the least schooling. We also observed a difference in the age distributions of syphilis seroreactivity (decreasing with age) and HIV (peaking at 26-30 years).

Data from Tanzania estimate that antenatal syphilis screening is still cost-effective in sub-Saharan Africa. Based on the 8\% RPR reactivity among ANW attendees in our study, the cost per disability adjusted life year averted by screening for syphilis (including stillbirths) at QECH would be about US $\$ 10^{9}$. In addition to the morbidity associated with adult syphilis infections, children born to syphilis infected mothers have higher morbidity rates ${ }^{10}$, and increased odds of stillbirth, neonatal death, and post-neonatal death ${ }^{11}$. Syphilis is a significant risk factor for HIV subtype $\mathrm{C}$ mother to child transmission $^{5}$, which suggests that treatment of maternal syphilis could also reduce pediatric HIV infections.

Antenatal care policy in Malawi states that all pregnant women should be screened for syphilis, and if RPR seroreactive, treated with benzathine penicillin. In our setting, syphilis seroreactive participants attended the ANC on average four times prior to study enrollment, which should have provided ample opportunity for syphilis screening and treatment early in pregnancy. Why the women were not previously tested for syphilis was not addressed by this study, nor was the possibility that the women were previously tested and treated. In order to promote optimal antenatal syphilis screening and treatment in Malawi, current routine practices and obstacles should be evaluated to minimize missed opportunities.

Previous studies in sub-Saharan Africa assessing the relationship between HIV and education level have produced conflicting results ${ }^{12,13}$. Two studies in Malawi, the 2003 Malawi NAC report and one by Taha and colleagues in $1998^{14}$, reported a decrease in HIV prevalence as the level of education increased; in contrast, our study found no such association. To rule out different definitions of education level, we stratified our education data into the 3 categories reported by Taha and colleagues, and still failed to observe an association between education and HIV status $(p=0.9)$. 
We are unable to conclude whether our results reflect an HIV epidemic that increasingly affects the more educated layers of society or whether the different observations might be a result of differences in sampling design or reflect variations in local HIV transmission dynamics.

In contrast to HIV, and consistent with other reports ${ }^{15-17}$, an increase in maternal education was independently associated with a decrease in syphilis seroreactivity. While the data presented here do not allow us to assess the underlying cause of this association, this finding should encourage the design of specific strategies and health education messages to curb syphilis and HIV-infection among women who attend the antenatal ward.

It has been reported elsewhere in sub-Saharan Africa that Muslims have lower HIV prevalence than non-Muslims ${ }^{18}$. According to the 2000 Malawi Demographic and Health Survey, $4 \%$ of non-Yao Malawians were Muslim while $75 \%$ of the Yao were Muslim (H. Misiri, unpublished observation). Because of their religious beliefs and according to anecdotal observations, it is likely that the Yao are more likely to routinely circumcise men than other tribes in Malawi. Male circumcision has recently been shown to protect men from incident $\mathrm{HIV}^{19-21}$, and it is therefore plausible to speculate that the lower prevalence among the Yao women is due to lower HIV prevalence among their circumcised partners ${ }^{22}$. However, because our questionnaire did not ascertain the partner's religion, the prevalence of partner circumcision, and other specific cultural practices, we cannot definitively determine if partner circumcision is associated with the low HIV prevalence among pregnant Yao women.

Our findings are limited by the cross-sectional nature of the data, the self-reported nature of many of the exposure variables, and our inability to accurately define active syphilis among the syphilis seroreactors. Approximately 38\% of the women asked to participate in this study declined, and because there were no additional data collected on those who refused, we are unsure how well these data can be generalized to the total population of women attending the ANC at Queen Elizabeth Central Hospital. Although we used a qualitative serology-based case definition of syphilis, which may give limited information in the absence of results of recent testing and treatment, our observations are still useful. While a high proportion of RPR reactive sera $(27 \%)$ were not confirmed by TPHA, the observed association between reported history of spontaneous abortion and RPR and TPHA seroreactivity gives credibility to the syphilis testing. Women found to be syphilis seroreactive represent women who should have been treated early during their pregnancy. Finally, because we enrolled women who presented to the antenatal ward at QECH, which is a tertiary care center, these results are likely not representative of the general Malawian population. Further studies assessing the socio-demographic correlates of HIV and syphilis infection in non-referral centers and in the community are warranted.

In conclusion, we report a 30\% HIV prevalence and a $5 \%$ syphilis seroreactivity among pregnant women who presented to the ANW at QECH. Although there was substantial overlap between the factors associated with HIV and syphilis seroreactivity, the two infections affect slightly different populations. Several initiatives have been taken to increase antiretroviral treatment coverage among pregnant Malawians for both HIV treatment and for MTCT prophylaxis (for example, see http://www.id.unc.edu/ malawi/studies_research.htm). These programs should be complemented with improved efforts in antenatal screening and treatment of syphilis ${ }^{23}$.

\section{Acknowledgements}

We thank the Malawian women who participated in this study. We thank Debbie Kamwendo, Patrick Mkundika (deceased), Benson Thindwa, and Visopo Harawa for their logistical and technical support; Audrey Pettifor for reading and providing helpful comments on the manuscript; and Ebbie Chalaluka and the study nurses for their invaluable contributions to subject recruitment, data collection, and patient care. This research was presented, in part, at both the 9th annual Malawi College of Medicine Research Dissemination Day and the 12th Annual Conference on Retroviruses and Opportunistic Infections.

\section{Funding}

Support for this research was provided by the University of North Carolina at Chapel Hill Center for AIDS Research (P30-AI50410) and the National Institutes of Health National Institute of Allergy and Infectious Disease (AI0715126, AI065369-01, and AI49084). MEM is supported by a Research Leave Fellowship and SJR is supported by a Senior Fellowship, both from The Wellcome Trust. The funding agencies had no role in study design, data collection, data analysis, decision to publish, or preparation of the manuscript.

\section{References}

1. National AIDS Commission: HIV/AIDS in Malawi: Estimates of the prevalence of infection and the implications. Ministry of Health and Population; 2003: 23.

2. Finelli L, Berman SM, Koumans EH, Levine WC: Congenital syphilis. Bull World Health Organ 1998, 76 Suppl 2:126-128.

3. Genc M, Ledger WJ: Syphilis in pregnancy. Sex Transm Infect 2000, 76(2):73-79.

4. Watson-Jones D, Changalucha J, Gumodoka B, Weiss H, Rusizoka M, Ndeki L, Whitehouse A, Balira R, Todd J, Ngeleja D et al: Syphilis in pregnancy in Tanzania. I. Impact of maternal syphilis on outcome of pregnancy. J Infect Dis 2002, 186(7):940-947.

5. Mwapasa V, Rogerson SJ, Kwiek JJ, Wilson PE, Milner D, Molyneux ME, Kamwendo DD, Tadesse E, Chaluluka E, Meshnick SR: Maternal syphilis infection is associated with increased risk of mother-to-child transmission of HIV in Malawi. Aids 2006, 20(14):1869-1877.

6. Gloyd S, Chai S, Mercer MA: Antenatal syphilis in sub-Saharan Africa: missed opportunities for mortality reduction. Health Policy Plan 2001, $16(1): 29-34$

7. van Eijk AM, Bles HM, Odhiambo F, Ayisi JG, Blokland IE, Rosen DH, Adazu K, Slutsker L, Lindblade KA: Use of antenatal services and delivery care among women in rural western Kenya; a community based survey. Reprod Health 2006, 3(1):2.

8. Bowie C, Kadzandira J, Mburu F, Mwapasa V, Singleton G: Second Interim Report (Jan-June 2005). National AIDS Programme: Independant Multi-Disciplinary Review Team; 2005.

9. Terris-Prestholt F, Watson-Jones D, Mugeye K, Kumaranayake L, Ndeki L, Weiss H, Changalucha J, Todd J, Lisekie F, Gumodoka B et al: Is antenatal syphilis screening still cost effective in sub-Saharan Africa. Sex Transm Infect 2003, 79(5):375-381.

10. Watson-Jones D, Oliff M, Terris-PrestholtF, Changalucha J, Gumodoka B, Mayaud P, Semakafu AM, Kumaranayake L, Gavyole A, Mabey D et al: Antenatal syphilis screening in sub-Saharan Africa: lessons learned from Tanzania. Trop Med Int Health 2005, 10(9):934-943.

11. McDermott J, Steketee R, Larsen S, Wirima J: Syphilis-associated perinatal and infant mortality in rural Malawi. Bull World Health Organ 1993, 71(6):773-780. 
12. Hargreaves JR, Glynn JR: Educational attainment and HIV-1 infection in developing countries: a systematic review. Trop Med Int Health 2002, 7(6):489-498.

13. Walque D, Nakiyingi-Miiro JS, Busingye J, Whitworth JA: Changing association between schooling levels and HIV-1 infection over 11 years in a rural population cohort in south-west Uganda. Trop Med Int Health 2005, 10(10):993-1001.

14. Taha TE, Dallabetta GA, Hoover DR, Chiphangwi JD, Mtimavalye LA, Liomba GN, Kumwenda NI, Miotti PG: Trends of HIV-1 and sexually transmitted diseases among pregnant and postpartum women in urban Malawi. Aids 1998, 12(2):197-203.

15. Potter D, Goldenberg RL, Read JS, Wang J, Hoffman IF, Saathoff E, Kafulafula G, Aboud S, Martinson FE, Dahab M et al: Correlates of Syphilis Seroreactivity Among Pregnant Women: The HIVNET 024 Trial in Malawi, Tanzania, and Zambia. Sex Transm Dis 2006.

16. Swai RO, Somi GR, Matee MI, Lyamuya EF, Killewo J, Kwesigabo G, Tulli T, Kabalimu TK, Ng'ang'a L, Isingo R et al: Surveillance of HIV and syphilis infections among antenatal clinic attendees In Tanzania2003/2004. BMC Public Health 2006, 6(1):91.

17. Behets FM AJ, Randrianasolo D, Randriamanga R, Rasamilalao D, Chen CY, Weiss JB, Morse SA, Dallabetta G, Cohen MS.: Chancroid, primary syphilis, genital herpes, and lymphogranuloma venereum in Antananarivo, Madagascar. Journal of Infectious Diseases 1999, 180(4):1382-1385.

\section{Edititured from page 77 Top 10 Picks}

\section{The impact of HIV on mortality during in- patient rehabilitation of severely malnourished children in Malawi.}

Chinkhumba J, Tomkins A, Banda T et al

Trans R Soc Trop Med Hyg. 2008;102(7):639-44.

A prospective cohort study measured mortality during nutritional rehabilitation among HIV-infected and uninfected children, aged 6-59 months, with severe acute malnutrition (SAM). The authors conclude that routine testing and treatment for HIV among all malnourished children is necessary to improve quality of care and reduce mortality among children with SAM.

\section{Outcomes in HIV-infected patients who develop tuberculosis after starting antiretroviral treatment in Malawi.}

Yu JK, Bong CN, Chen SC et al

Int J Tuberc Lung Dis. 2008;12(6):692-4.

A retrospective review was conducted of patients starting antiretroviral treatment (ART) at Mzuzu Central Hospital, Malawi, to identify those who developed tuberculosis (TB) within 6 months of commencing ART and document their treatment outcomes. The authors propose easier methods are needed to diagnose TB in human immunodeficiency virusinfected patients and to prevent patients from developing TB while on ART.

\section{Natural history and risk factors associated with early and established HIV type 1 infection among reproductive-age women in Malawi.}

Kumwenda JJ, Makanani B, Taulo F et al

Clin Infect Dis. 2008 15;46(12):1913-20.
18. Gray PB: HIV and Islam: is HIV prevalence lower among Muslims? Soc Sci Med 2004, 58(9):1751-1756.

19. Auvert B, Taljaard D, Lagarde E, Sobngwi-Tambekou J, Sitta R, Puren A: Randomized, Controlled Intervention Trial of Male Circumcision for Reduction of HIV Infection Risk: The ANRS 1265 Trial. PLoS Med 2005, 2(11):e298.

20. Bailey RC, Moses S, Parker CB, Agot K, Maclean I, Krieger JN, Williams CF, Campbell RT, Ndinya-Achola JO: Male circumcision for HIV prevention in young men in Kisumu, Kenya: a randomised controlled trial. Lancet 2007, 369(9562):643-656.

21. Gray RH, Kigozi G, Serwadda D, Makumbi F, Watya S, Nalugoda F, Kiwanuka N, Moulton LH, Chaudhary MA, Chen MZ et al: Male circumcision for HIV prevention in men in Rakai, Uganda: a randomised trial. Lancet 2007, 369(9562):657-666.

22. Gray R, Wawer MJ, Thoma M, Serwadda D, Nalugoda F, Li X, Kigozi G, Kiwanuka N, Laeyendecker O, Quinn T: Male Circumcision and the Risks of Female HIV and Sexually Transmitted Infections Acquisition in Rakai, Uganda. In: 13th Conference on Retroviruses and Opportunistic Infections. Denver, CO; 2006.

23. Peeling RW, Mabey D, Fitzgerald DW, Watson-Jones D: Avoiding HIV and dying of syphilis. Lancet 2004, 364(9445):1561-1563.

The study examines plasma viral levels and trends during early and established HIV-1 infection among reproductiveage women who participated in a randomized trial to treat genital tract infection in Malawi. Also assessed was the association of injectable hormonal contraceptive use with HIV-1 infection. The authors conclude that knowledge of virus set point and trends of viral load in HIV-1 seroincident and seroprevalent asymptomatic women could assist in antiretroviral treatment management.

\section{Causes of blindness among children identified through village key informants in Malawi.}

Kalua K, Patel D, Muhit M, Courtright P

Can J Ophthalmol. 2008;43(4):425-7.

A cross sectional study of children in communities of Chikwawa district, Malawi was conducted to determine the causes of blindness among children in rural Malawi.. Cataract (congenital/developmental) was the leading cause of blindness (35\%), followed by corneal conditions $(22 \%)$. The authors propose that this Chikwakwa is an area once noted for vitamin A deficiency in children resulting in cataract development. Priorities for early cataract detection and intervention need to be considered for restoration of sight in children.

\section{Nutritional status at admission of children with cancer in Malawi.}

Israëls T, Chirambo C, Caron HN, Molyneux EM

Pediatr Blood Cancer. 2008;51(5):626-8.

Arm anthropometry shows that more than half of Malawian children with cancer are severely acutely malnourished at diagnosis. Weight for height, in children with large tumour masses, is less sensitive than arm anthropometry in detecting acute malnutrition. Forty-five percent of paediatric oncology patients in Malawi are stunted, making interpretation of weight for age very difficult. 